\title{
Reversing Quantum Trajectories with Analog Feedback
}

\author{
G. de Lange, ${ }^{1}$ D. Ristè,${ }^{1}$ M. J. Tiggelman, ${ }^{1}$ C. Eichler, ${ }^{2}$ L. Tornberg, ${ }^{3}$ G. Johansson, ${ }^{3}$ A. Wallraff, ${ }^{2}$ \\ R. N. Schouten, ${ }^{1}$ and L. DiCarlo ${ }^{1}$ \\ ${ }^{1}$ Kavli Institute of Nanoscience, Delft University of Technology, P.O. Box 5046, 2600 GA Delft, Netherlands \\ ${ }^{2}$ Department of Physics, ETH Zürich, CH-8093 Zürich, Switzerland \\ ${ }^{3}$ Department of Microtechnology and Nanoscience, MC2, Chalmers University of Technology, SE-412 96 Gothenburg, Sweden
} (Received 22 November 2013; published 24 February 2014)

\begin{abstract}
We demonstrate the active suppression of transmon qubit dephasing induced by dispersive measurement, using parametric amplification and analog feedback. By real-time processing of the homodyne record, the feedback controller reverts the stochastic quantum phase kick imparted by the measurement on the qubit. The feedback operation matches a model of quantum trajectories with a measurement efficiency $\tilde{\eta} \approx 0.5$, consistent with the result obtained by postselection. We overcome the bandwidth limitations of the amplification chain by numerically optimizing the signal processing in the feedback loop and provide a theoretical model explaining the optimization result.
\end{abstract}

PACS numbers: 03.67.Lx, 42.50.Dv, 42.50.Pq, 85.25.-j

In a quantum measurement, information gain is accompanied by backaction, altering superposition states of the observed system [1]. Tunable strength measurements have been devised to balance the tradeoff between information gain and backaction. These can be realized, for example, by controlling the interaction of the observed qubit with an ancillary qubit, followed by strong measurement of the ancilla [2-4]. Depending on the choice of ancilla measurement basis, the observed qubit either acquires a stochastic phase kick, or is partially projected towards one of the basis states, in a direction that is determined by the measurement result. Similarly, a cavity mode can serve as an ancilla, with the measurement basis set by the detected field quadrature [5], and a continuous range of measurement results and associated kickbacks [5,6].

For an efficient measurement [1], the correlation between the stochastic evolution of the system, also known as quantum trajectory, and the measurement record of the ancilla can be exploited to undo any unwanted backaction $[7,8]$ or to reverse the measurement altogether [9]. Probabilistic reversal of measurement backaction has been pursued with superconducting [10], photonic [11], and ionic systems [12]. Deterministic reversal, requiring feedback control, has only been demonstrated with ions [13]. Recent improvements in quantum coherence in circuit quantum electrodynamics (cQED) [14] have allowed first demonstrations of feedback control with superconducting qubits. Digital feedback, based on fully projective measurement, enabled on-demand qubit state initialization [15,16], deterministic teleportation [17], and generation of deterministic entanglement by parity measurement [18]. Analog feedback, instead, is required to counteract the continuous range of measurement kickbacks in a qubit-cavity system. A first implementation of analog feedback relied on continuous monitoring of a driven qubit to stabilize Rabi oscillations [19].
In this Letter, we demonstrate the real-time reversal of measurement-induced qubit dephasing in cQED, using phase-sensitive parametric amplification [20] and analog feedback control, as proposed in Ref. [21]. The recovery of coherence by feedback is quantitatively consistent with a measurement efficiency $\tilde{\eta} \approx 0.5$ for the homodyne detection chain, closely matching the result obtained by open-loop postselection. Furthermore, we demonstrate a numerical procedure that finds the optimal weight function for the homodyne signal integration, circumventing the inefficiency arising from the finite detection bandwidth.

We study measurement-induced dephasing of a transmon qubit (transition frequency $\omega_{Q} / 2 \pi=5.430 \mathrm{GHz}$ ) coupled to the fundamental mode of a 3D cavity (frequency $f_{r}=6.5433 \mathrm{GHz}$, linewidth $\kappa / 2 \pi=1.4 \mathrm{MHz}$ ). The qubitcavity Hamiltonian in the presence of a measurement drive at frequency $f_{m}$ and valid in the dispersive regime of our experiment is [22]

$$
H=\left(\Delta_{r}-\chi Z\right) a^{\dagger} a-\omega_{Q} Z / 2+\epsilon_{m}(t) a+\epsilon_{m}^{*}(t) a^{\dagger},
$$

in a frame rotating at $f_{m}$, with $\Delta_{r} / 2 \pi=f_{r}-f_{m}, a\left(a^{\dagger}\right)$ the photon annihilation (creation) operator, and $Z$ the qubit Pauli $z$ operator. Above, we have grouped terms to highlight the dependence of the cavity resonance on the qubit state. The transmitted signal is sent to a Josephson parametric amplifier (JPA) operated in phase-sensitive mode $[20,23]$. The homodyne signal obtained by demodulation is recorded for postprocessing purposes and also sampled by a feedback controller implementing real-time phase correction (discussed further below) [Fig. 1(a)]. We choose for $f_{m}$ the average of the cavity frequencies for the qubit in $|0\rangle$ $\left(f_{r}\right)$ and $|1\rangle\left(f_{r}+\chi / \pi\right.$, with $\left.\chi / \pi=-3.2 \mathrm{MHz}\right)$ [Fig. 1(b)].

Applying a measurement pulse entangles the qubit with the cavity field $[6,24]$. If the measurement record is 

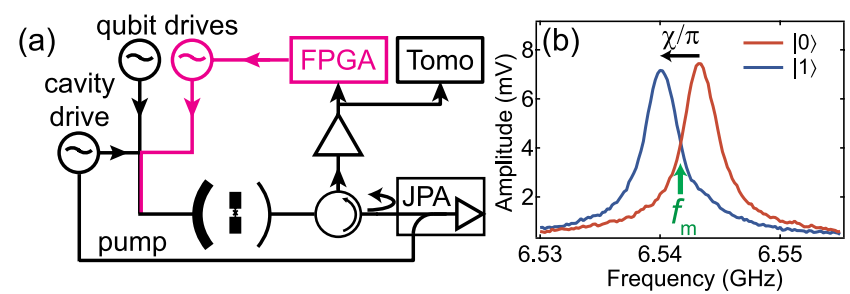

(c)

(d)

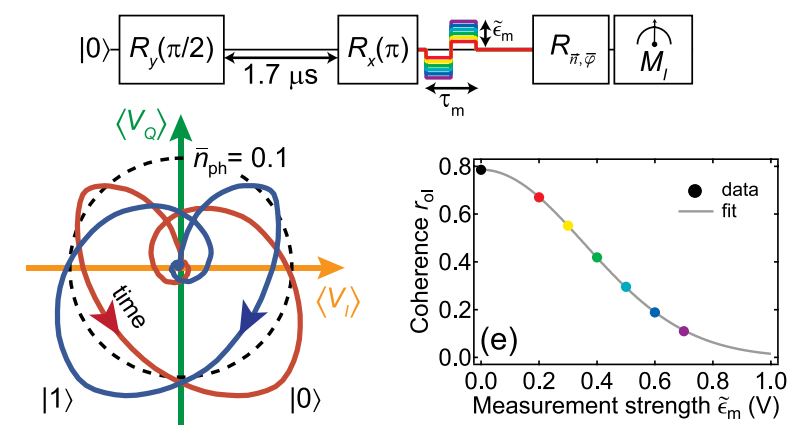

FIG. 1 (color online). Measurement-induced dephasing and analog feedback scheme. (a) Diagram of the key elements of the experimental setup. Qubit measurement and control drives are coupled to the input port of an asymmetrically coupled 3D cavity $\left(\kappa_{\text {in }} / \kappa_{\text {out }} \approx 1 / 30\right)$. The signal emitted at the output port is added to the pump tone, which biases the JPA to a voltage gain $G=16$ and a bandwidth $\kappa_{\mathrm{JPA}} / 2 \pi=5.7 \mathrm{MHz}$ (Fig. S7). The reflected, amplified signal $[20,23]$ is directed by a circulator to a semiconductor amplifier (HEMT) at $3 \mathrm{~K}$. At room temperature, the signal is split into two arms, one for data acquisition and another feeding the FPGA-based feedback controller (see Fig. S1 for setup details). (b) Cavity spectroscopy for the qubit prepared in $|0\rangle$ and $|1\rangle$. Measurement pulses are applied at $f_{m}$ (green arrow). (c) Echo sequence, where in the second half a measurement pulse with amplitude $\tilde{\epsilon}_{m}$ is inserted to study its dephasing effect on the qubit. The second $\pi / 2$ pulse is compiled into the tomographic rotation $R_{\vec{n}, \bar{\varphi}}$, where $R_{\vec{n}}$ is either $R_{y}(-\pi / 2), R_{x}(\pi / 2)$ or $I$, and the axis is rotated by $\bar{\varphi}$ around $z$ to cancel the deterministic phase shift. (d) Parametric plot of the averaged homodyne response $\left\langle V_{Q}\right\rangle$ versus $\left\langle V_{I}\right\rangle$ for measurement phase $\phi=\pi / 2$ and 0 , respectively, for the qubit in $|0\rangle$ (red) and $|1\rangle$ (blue), with $\tilde{\epsilon}_{m}=0.4 \mathrm{~V}$. Dashed circle: signal corresponding to $\bar{n}_{\mathrm{ph}}=0.1$ intracavity average photon. (e) Qubit coherence $r_{\mathrm{ol}}$ as a function of $\tilde{\epsilon}_{m}$. The best-fit curve gives the lever arm $\epsilon_{m} / \tilde{\epsilon}_{m}=$ $2 \pi \times 1.2 \mathrm{MHz} / \mathrm{V}$. For $\tilde{\epsilon}_{m}=0, r_{\mathrm{ol}}=r_{\text {off }}=0.79 \pm 0.01$.

disregarded, the absolute qubit coherence $r=\left|\rho_{01}\right|$ is reduced, where $\rho_{01}=\langle 0|\rho| 1\rangle$ is the off-diagonal element of the qubit density matrix. We observe this effect by applying a pulsed measurement drive with the qubit ideally starting in the superposition state $(|0\rangle+|1\rangle) / \sqrt{2}$. The measurement pulse is applied during the second half of an echo sequence [Fig. 1(c)], preferred over a Ramsey sequence to reduce the dephasing from mechanisms not inherent to the applied measurement. The pulse envelope has magnitude $\tilde{\epsilon}_{m}$ and the sign reversed halfway during the total duration of $500 \mathrm{~ns}$. The measured and amplified quadrature of the cavity response is set by the phase $\phi$ between the measurement pulse and the JPA pump.
In particular, for $\phi=0$, the averaged homodyne response is equal and opposite for the qubit in $|0\rangle$ and $|1\rangle$, $\left\langle V_{I}\right\rangle_{0}=-\left\langle V_{I}\right\rangle_{1}$, whereas for $\phi=\pi / 2,\left\langle V_{Q}\right\rangle_{0}=\left\langle V_{Q}\right\rangle_{1}$ [Figs. 1(d), S2] [25]. The measurement reduces $r_{\text {off }}$, the qubit coherence at the end of the echo sequence for $\tilde{\epsilon}_{m}=0$, to the open-loop coherence $r_{\mathrm{ol}}$. According to theory [26], $r_{\mathrm{ol}}=r_{\mathrm{off}} \exp \left[-\int_{0}^{t} \Gamma_{d}(\tau) d \tau\right]$, with instantaneous measurement-induced dephasing rate $\Gamma_{d}(t)=2 \chi \operatorname{Im}\left[\alpha_{0}(t) \alpha_{1}^{*}(t)\right]$, where $\alpha_{i}=\langle a\rangle_{i} \propto \epsilon_{m}$ is the complex-valued intracavity field for the qubit in $|i\rangle$. As expected, we observe a Gaussian decay of $r_{\mathrm{ol}}$ as a function of $\tilde{\epsilon}_{m}$ [Fig. 1(e)]. Note that $\Gamma_{d}$ is independent of $\varphi$ (data not shown) $[27,28]$.

Collecting the field emitted by the cavity during a measurement reveals the quantum trajectory followed by the qubit. The measurement basis and the corresponding kickback on the qubit depend on the choice of $\phi[27,28]$. The $\phi$-specific backaction becomes evident by conditioning (binning) the tomography results $M_{I}$ on the processed homodyne voltage. As first demonstrated in Ref. [5], for $\phi=0$, the measurement discriminates between qubit states and coherence is lost by gradual projection to the north or south pole of the Bloch sphere (Fig. S3 [25]). For $\phi=\pi / 2$, the case we focus on here, the measurement does not discriminate between qubit states and the kickback is a stochastic azimuthal phase $\delta \phi$ ( $z$ rotation). According to theory for a detector with infinite bandwidth [21,27,29], this phase depends on the integrated weighted homodyne voltage $V_{\text {int }}=\int w(t) V_{Q}(t) \mathrm{d} t$, with the weight function $w(t) \propto \operatorname{Re}\left[\alpha_{0}(t)\right] / \epsilon_{m}$, as

$$
\rho_{01}\left(V_{\text {int }}\right)=r_{\text {off }} \exp \left[(\eta-1) \int \Gamma_{d}(t) d t+i \varphi\right],
$$

where $\varphi=c V_{\text {int }}+\bar{\varphi}$, with $c \propto \epsilon_{m}$ and $\bar{\varphi}$ the deterministic ac-Stark phase shift [26]. Here, $\eta$ is the quantum efficiency, modeled as losses in the readout chain leading up to the JPA. In our experiment, the zero-average envelope of the measurement pulse, which makes $\int w(t) d t=0$, is chosen to suppress the infiltration of excess low-frequency noise in $V_{\text {int }}$ [30]. Furthermore, the integration window extends $6.5 / \kappa=0.75 \mu$ s past the end of the applied measurement pulse [Fig. 2(a)] in order to capture the total field emitted by the cavity as it returns to the vacuum state [21]. Binning the tomography results $M_{I}$ on $V_{\text {int }}$ reveals the stochastic phase $\delta \varphi$ induced by the measurement [Figs. 2(b)-2(d)] [5]. Rather than relying on the weight function predicted by theory, we numerically optimize $w=w_{\text {opt }}$ to maximize the conditioned coherence $r_{\text {con }}=\sum C\left(V_{\text {int }}\right) r\left(V_{\text {int }}\right)$, with $r$ the absolute coherence and $C$ the fraction of counts for the bin centered at $V_{\text {int }}$ [25]. From the conditioned coherence, we place a lower bound on $\eta$, absorbing signal losses after the JPA and classical processing of $V_{Q}$ in an overall measurement efficiency $\tilde{\eta}$ in Eq. (1). We find quantitative agreement with the data for $\tilde{\eta}=0.50$ [Figs. 2(c)-2(d)]. 


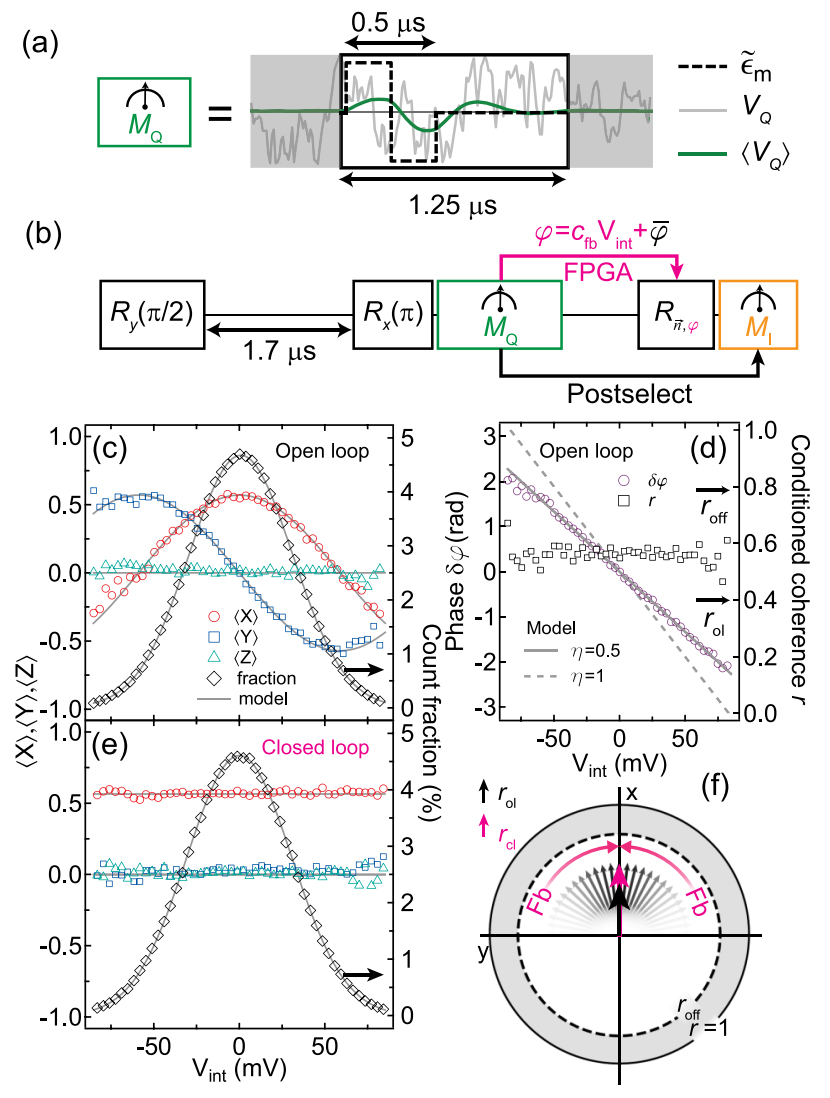

FIG. 2 (color online). Conditional qubit tomography and cancellation of measurement-induced dephasing by analog feedback. (a) The measurement $M_{Q}$ is performed with a pulse at $f_{m}$ with amplitude $\tilde{\epsilon}_{m}=0.4 \mathrm{~V}$ and $500 \mathrm{~ns}$ length (dashed trace). The homodyne record $V_{Q}$ is acquired for a total duration of $1.25 \mu \mathrm{s}$ from the start of the measurement pulse. Light (dark) trace: single (average) record. (b) Measurement scheme. (c) Conditional state tomography (left) and corresponding fraction of counts $C$ (right) in open-loop operation. Solid (dashed) curves: data (model with $\tilde{\eta}=0.50)$. The tomography outcomes $M_{I}$ are binned on $V_{\text {int }}=\sum_{n} w[n] V_{Q}[n]$, where $V_{Q}$ is sampled every $10 \mathrm{~ns}$. The weight function $w=w_{\text {opt }}$ is obtained by numerical optimization using the records $V_{Q}$ (see also Fig. 4. (d) Stochastic qubit phase $\delta \varphi$ (dots) and absolute coherence $r$ (squares), binned on $V_{\text {int }}$, and model for $\delta \varphi$ with $\tilde{\eta}=0.50$ (solid) and 1 (dashed line). In closedloop operation [(e), corresponding to $c_{\mathrm{fb}}=-10$ in Fig. 3(a)], $V_{Q}$ is fed to the feedback controller, which calculates $V_{\text {int }}$ using $w_{\text {opt }}$ and translates it into $\delta \varphi$, setting the phase of $R_{\vec{n}, \varphi}$. (f) Measured distribution of $\delta \varphi$ (grey scale) produced by $M_{Q}$ and refocusing by analog feedback. This refocusing increases the unconditioned coherence from $r_{\mathrm{ol}}=0.40$ (black arrow) to $r_{\mathrm{cl}}=0.56$ (pink arrow). Dashed circle: maximum $r=r_{\text {off }}$ that would be obtained with $\tilde{\eta}=1$.

Moving beyond postselection, we now set off to cancel the measurement-induced kickback by employing analog feedback control. In real time, the controller samples $V_{Q}$, calculates $V_{\text {int }}$ using $w_{\text {opt }}$, and adjusts the phase of the tomographic prerotation $R_{\vec{n}, \phi}$ by $\delta \varphi=c_{\mathrm{fb}} V_{\text {int }}$ (Figs. S4, S5 [25]). The optimal choice for the feedback gain $\left(c_{\mathrm{fb}}=c_{\mathrm{opt}}\right)$ removes all the azimuthal phase dependence on $V_{\text {int }}$ (a)

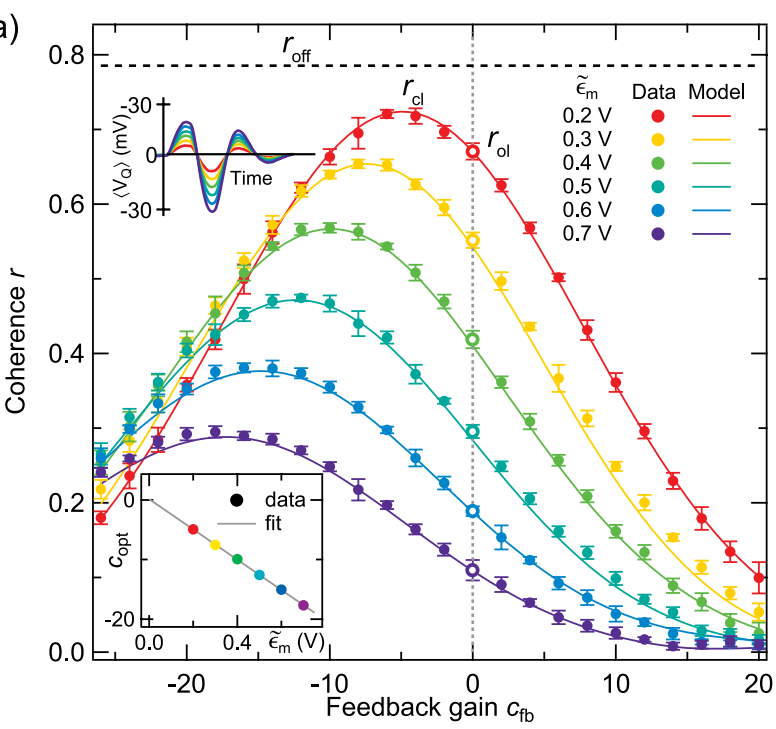

(b)

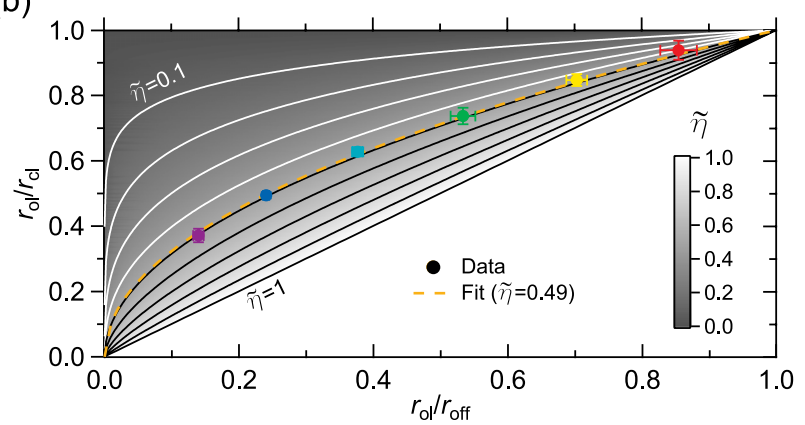

FIG. 3 (color online). Extraction of measurement efficiency from the extent of coherence recovery. (a) Coherence versus feedback gain $c_{\mathrm{fb}}$ for $\tilde{\epsilon}_{m}=0.2-0.7 \mathrm{~V}$, with $w_{\text {opt }}$ optimized at $\tilde{\epsilon}_{m}=0.4 \mathrm{~V}$. Top left: average homodyne voltage $\left\langle V_{Q}\right\rangle$ for the same range of $\tilde{\epsilon}_{m}$. The maximum coherence $r_{\mathrm{cl}}$ corresponds to the optimum feedback gain $c_{\text {opt }}$ (lower inset), directly proportional to $\tilde{\epsilon}_{m}$. The horizontal dashed line indicates the coherence $r_{\text {off }}$ for no measurement drive $\left(\tilde{\epsilon}_{m}=0\right)$. Error bars are the standard deviations of eight repetitions. (b) Contour plot of the measurement efficiency $\tilde{\eta}$, with curves at 0.1 steps. For each $\tilde{\epsilon}_{m}, r_{\mathrm{cl}}$ is obtained by a quadratic fit of $r$ around the maximum and $r_{\mathrm{ol}}$ is the measured average for $c_{\mathrm{fb}}=0$ in (a). The best fit of Eq. (2) (orange dashed line) to the data yields $\tilde{\eta}=0.49 \pm 0.01$.

[Figs. 2(e)-2(f)]. Crucially, $r_{\text {con }}$ is unaffected, demonstrating that feedback does not introduce additional errors.

To fully quantify the performance of the active coherence recovery, we repeat the experiment in Fig. 2(b) for various measurement-drive amplitudes $\tilde{\epsilon}_{m}$ and feedback gains $c_{\mathrm{fb}}$ [Fig. 3(a)]. Whereas the variance of $V_{\text {int }}$ is independent of $\tilde{\epsilon}_{m}$, as expected, the phase dependence $\mathrm{d} \delta \phi / \mathrm{d} V_{\text {int }}$ grows linearly with $\tilde{\epsilon}_{m}[27,29]$, requiring the optimum $c_{\text {opt }} \propto \tilde{\epsilon}_{m}$ [Fig. 3(a) inset]. Following from Eq. (1), the measured $r_{\mathrm{ol}}$ (corresponding to $\left.c_{\mathrm{fb}}=0\right), r_{\text {off }}\left(\tilde{\epsilon}_{m}=0\right)$ and $r_{\mathrm{cl}}\left(c_{\mathrm{fb}}=c_{\mathrm{opt}}\right)$ are related by

$$
r_{\mathrm{ol}} / r_{\mathrm{cl}}=\left(r_{\mathrm{ol}} / r_{\mathrm{off}}\right)^{\tilde{\eta}}
$$

We obtain the best-fit $\tilde{\eta}=0.49 \pm 0.01$ [Fig. 3(b)]. 

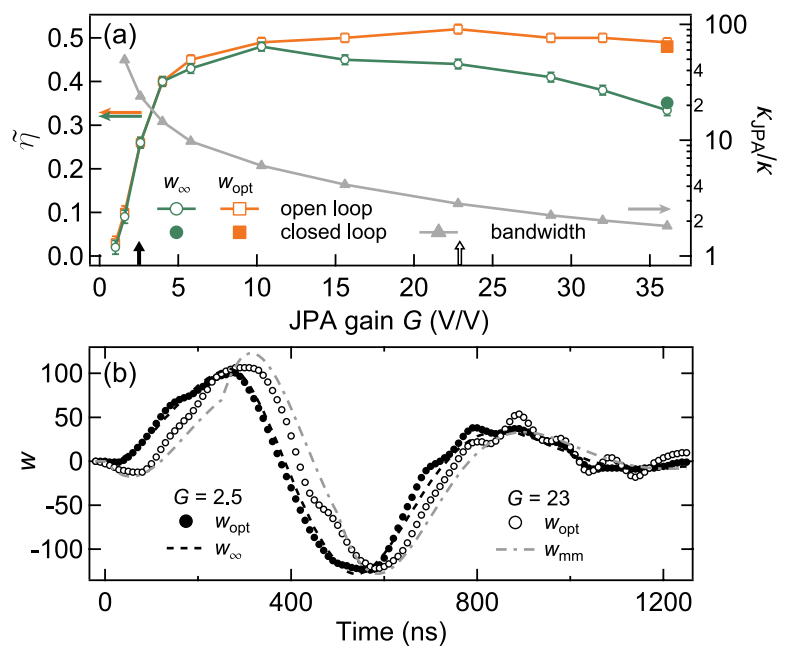

FIG. 4 (color online). (a) Measurement efficiency as a function of JPA gain and bandwidth. The experiment in Fig. 2(b) is repeated for different pump powers (with the JPA resonance kept at $f_{m}$ ), setting the JPA voltage gain $G$ and bandwidth $\kappa_{\text {JPA }}$ (triangles). For $G \lesssim 10$, the gain is insufficient to overcome the noise temperature of the HEMT (noise temperature $\sim 3 \mathrm{~K}$ ). For higher $G$, the infinite-bandwidth $w_{\infty}$ becomes suboptimal as $V_{Q}$ is low-pass filtered by the JPA when $\kappa_{\mathrm{JPA}}$ approaches $\kappa$ (dots). By numerically optimizing the weight function $\left(w_{\text {opt }}\right)$, this filtering is undone and $\tilde{\eta} \approx 0.5$ is recovered (squares). For $G=1$ the pump is turned off and the JPA is intentionally detuned by $\sim 200 \mathrm{MHz}$ from $f_{m}$. (b) Numerically optimized $w_{\text {opt }}$ for $G=2.5$ (full dots) and 23 (empty dots), model $w_{\infty}$ for infinite detector bandwidth (dashed line) and mode-matched $w_{\mathrm{mm}}$ (dot-dashed line) for $\kappa_{\mathrm{JPA}} / 2 \pi=3.9 \mathrm{MHz}(G=23)$.

Finally, we investigate the influence of detection settings on $\tilde{\eta}$. By adjusting the pump power, we tune the JPA voltage gain $G$ and bandwidth $\kappa_{\mathrm{JPA}}$, their product being roughly constant at $\sim 90 \mathrm{MHz}$ (Fig. S6) [20,25]. For each setting, we perform conditional tomography (as in Fig. 2) and extract $\tilde{\eta}$ using Eq. (2). In a first approach, we use the predicted $[21,25,31]$ weight function $w_{\infty} \propto \operatorname{Re}\left[\alpha_{0}\right]$ for infinite-bandwidth detection and unit gain [Fig. 4(a), dots]. For decreasing gain $(G<10), V_{Q}$ is not sufficiently amplified above the noise floor of the second amplification stage at $3 \mathrm{~K}$, causing $\tilde{\eta}$ to plummet. Increasing $G$ overcomes the noise floor at the expense of lowering $\kappa_{\mathrm{JPA}}$. However, for $G>10$, where $\kappa_{\mathrm{JPA}} \lesssim 4 \kappa$, the infinitebandwidth approximation no longer holds, resulting in a lower $\tilde{\eta}$. In a second approach, we run the numerical optimization procedure to determine $w_{\text {opt }}$ at each JPA setting (Fig. S6) [25]. In this way, we recover $\tilde{\eta} \approx 0.5$ even as $\kappa_{\mathrm{JPA}}$ approaches $\kappa$. This independence of $\tilde{\eta}$ at high $G$ suggests that inefficiency arises from microwave loss between the cavity and the JPA, as assumed by the model. The compensation for the finite detection bandwidth is reflected by the change of $w_{\text {opt }}$ with $\kappa_{\mathrm{JPA}}$ [Fig. 4(b)]. For $\kappa_{\mathrm{JPA}} \gg \kappa, w_{\text {opt }}$ closely matches $w_{\infty}$. For $\kappa_{\mathrm{JPA}} \approx \kappa$, instead, $w_{\text {opt }}$ differs significantly.
To understand how the JPA response impacts $w_{\text {opt }}$, we apply the recent mode-matching theory of Ref. [31]. This theory predicts the optimum weight function $w_{\mathrm{mm}} \propto\left\langle b_{\text {out }}^{\dagger}(t) Z\right\rangle$, with $b_{\text {out }}^{\dagger}(t)$ the operator for the outgoing field after amplification by the JPA [25]. As shown in the Supplemental Material [25], $w_{\text {mm }} \propto \mathcal{F}^{-1}\left[\left(\alpha_{0, \Delta}^{*}-\alpha_{1, \Delta}^{*}\right) / 2 G_{s, \Delta}\right]$, where $\alpha_{i, \Delta}=\left\langle a_{\Delta}\right\rangle_{i}$ for the qubit in $|i\rangle$, with $a_{\Delta}$ the Fourier component of the intracavity field at detuning $\Delta$ from the pump, $G_{s, \Delta}$ the $\Delta$-dependent small-signal gain, and $\mathcal{F}$ the Fourier transform. Interestingly, $w_{\mathrm{mm}}$ coincides with the expected $\left\langle V_{I}\right\rangle$ for the qubit in $|0\rangle$, corresponding to the quadrature deamplified by the JPA for $\phi=\pi / 2$. We find a good agreement between the predicted $w_{\mathrm{mm}}$ and the experimental $w_{\text {opt }}$ [Fig. 4(b)].

In conclusion, we demonstrated the suppression of measurement-induced dephasing of a transmon qubit using parametric amplification and analog feedback. Optimal real-time processing of the homodyne signal makes the recovery of coherence independent of detection bandwidth and equal to the maximum achievable with the quantum efficiency $\approx 0.5$. We estimate that applying the same feedback scheme to the cavity-assisted parity measurement $[29,32]$ in the same conditions as Ref. [18] would improve concurrence from the measured $34 \%$ to $42 \%$.

Improving quantum efficiency will be essential to fully undo measurement kickback and for protocols, such as qubit-state stabilization [33,34] and continuous-time error correction [35], requiring near-perfect correlation between measurement record and kickback. Alternatively, analog feedback schemes that rely on qubit projection can tolerate a lower efficiency, since estimation of the quantum state improves with the measurement strength. Similarly to the first implementations of digital feedback in the solid state [15-18], which reached high fidelity in spite of moderate efficiencies, analog feedback using projective measurement offers the capability to create and stabilize entanglement $[36,37]$ with the current state of the art.

We thank C. A. Watson for experimental assistance, W. F. Kindel and K. W. Lehnert for the parametric amplifier, and A.F. Kockum and M. Dukalski for helpful discussions. We acknowledge funding from the Dutch Organization for Fundamental Research on Matter (FOM), the Netherlands Organization for Scientific Research (NWO, VIDI scheme), and the EU FP7 integrated projects SOLID and SCALEQIT. G. d. L. and D. R. contributed equally to this work.

[1] H. M. Wiseman and G. J. Milburn, Quantum Measurement and Control (Cambridge University Press, Cambridge, England, 2009).

[2] G. J. Pryde, J. L. O'Brien, A. G. White, S. D. Bartlett, and T. C. Ralph, Phys. Rev. Lett. 92, 190402 (2004).

[3] J. P. Groen, D. Ristè, L. Tornberg, J. Cramer, P. C. de Groot, T. Picot, G. Johansson, and L. DiCarlo, Phys. Rev. Lett. 111, 090506 (2013). 
[4] M. S. Blok, C. Bonato, M. L. Markham, D. J. Twitchen, V. V. Dobrovitski, and R. Hanson, arXiv:1311.2899.

[5] K. W. Murch, S. J. Weber, C. Macklin, and I. Siddiqi, Nature (London), 502, 211 (2013).

[6] M. Hatridge et al., Science, 339, 178 (2013).

[7] H. M. Wiseman, Phys. Rev. A 51, 2459 (1995).

[8] A. N. Korotkov, Phys. Rev. B 71, 201305 (2005).

[9] A. N. Korotkov and A. N. Jordan, Phys. Rev. Lett. 97, 166805 (2006).

[10] N. Katz et al., Phys. Rev. Lett. 101, 200401 (2008).

[11] Y.-S. Kim, Y.-W. Cho, Y.-S. Ra, and Y.-H. Kim, Opt. Express 17, 11978 (2009).

[12] J. A. Sherman, M. J. Curtis, D. J. Szwer, D. T. C. Allcock, G. Imreh, D. M. Lucas, and A. M. Steane, Phys. Rev. Lett. 111, 180501 (2013).

[13] P. Schindler, T. Monz, D. Nigg, J. T. Barreiro, E. A. Martinez, M. F. Brandl, M. Chwalla, M. Hennrich, and R. Blatt, Phys. Rev. Lett. 110, 070403 (2013).

[14] M. H. Devoret and R. J. Schoelkopf, Science 339, 1169 (2013).

[15] D. Ristè, C. C. Bultink, K. W. Lehnert, and L. DiCarlo, Phys. Rev. Lett. 109, 240502 (2012).

[16] P. Campagne-Ibarcq, E. Flurin, N. Roch, D. Darson, P. Morfin, M. Mirrahimi, M. H. Devoret, F. Mallet, and B. Huard, Phys. Rev. X 3, 021008 (2013).

[17] L. Steffen, Y. Salathe, M. Oppliger, P. Kurpiers, M. Baur, C. Lang, C. Eichler, G. Puebla-Hellmann, A. Fedorov, and A. Wallraff, Nature (London) 500, 319 (2013).

[18] D. Ristè, M. Dukalski, C. A. Watson, G. de Lange, M. J. Tiggelman, Ya. M. Blanter, K. W. Lehnert, R. N. Schouten, and L. DiCarlo, Nature (London) 502, 350 (2013).

[19] R. Vijay, C. Macklin, D. H. Slichter, K. W. Murch, R. Naik, A. N. Korotkov, and I. Siddiqi, Nature (London) 490, 77 (2012).

[20] M. A. Castellanos-Beltran, K. D. Irwin, G. C. Hilton, L. R. Vale, and K. W. Lehnert, Nat. Phys. 4, 929 (2008).

[21] A. Frisk Kockum, L. Tornberg, and G. Johansson, Phys. Rev. A 85, 052318 (2012).
[22] A. Blais, R.-S. Huang, A. Wallraff, S. M. Girvin, and R. J. Schoelkopf, Phys. Rev. A 69, 062320 (2004).

[23] D. Ristè, J. G. van Leeuwen, H.-S. Ku, K. W. Lehnert, and L. DiCarlo, Phys. Rev. Lett. 109, 050507 (2012).

[24] C. Eichler, D. Bozyigit, and A. Wallraff, Phys. Rev. A 86, 032106 (2012).

[25] See Supplemental Material at http://link.aps.org/ supplemental/10.1103/PhysRevLett.112.080501 for device details, experimental methods, extended results and modeling for the optimum weight function.

[26] J. Gambetta, A. Blais, D. I. Schuster, A. Wallraff, L. Frunzio, J. Majer, M. H. Devoret, S. M. Girvin, and R. J. Schoelkopf, Phys. Rev. A 74, 042318 (2006).

[27] J. Gambetta, A. Blais, M. Boissonneault, A. A. Houck, D. I. Schuster, and S. M. Girvin, Phys. Rev. A 77, 012112 (2008).

[28] A. N. Korotkov, arXiv:1111.4016. Proceedings of Les Houches Summer School, "Quantum Machines" (2011).

[29] L. Tornberg and G. Johansson, Phys. Rev. A 82, 012329 (2010).

[30] With a square envelope, instead, we obtain $\tilde{\eta}=0.39$ using Eq. (3).

[31] C. Eichler, Ph.D. Dissertation, ETH Zurich, 2013.

[32] K. Lalumiére, J. M. Gambetta, and A. Blais, Phys. Rev. A 81, 040301 (2010).

[33] J. Wang and H. M. Wiseman, Phys. Rev. A 64, 063810 (2001).

[34] G. G. Gillett, R. B. Dalton, B. P. Lanyon, M. P. Almeida, M. Barbieri, G. J. Pryde, J. L. O’Brien, K. J. Resch, S. D. Bartlett, and A. G. White, Phys. Rev. Lett. 104, 080503 (2010).

[35] C. Ahn, A. C. Doherty, and A. J. Landahl, Phys. Rev. A 65 , 042301 (2002).

[36] M. Sarovar, H.-S. Goan, T. P. Spiller, and G. J. Milburn, Phys. Rev. A 72, 062327 (2005).

[37] Z. Liu, L. Kuang, K. Hu, L. Xu, S. Wei, L. Guo, and X.-Q. Li, Phys. Rev. A 82, 032335 (2010). 